TRANSACTIONS OF THE

AMERICAN MATHEMATICAL SOCIETY

Volume 359, Number 9, September 2007, Pages 4339-4358

S 0002-9947(07)04150-5

Article electronically published on March 20, 2007

\title{
SPECTRAL ZETA FUNCTIONS OF FRACTALS AND THE COMPLEX DYNAMICS OF POLYNOMIALS
}

\author{
ALEXANDER TEPLYAEV
}

\begin{abstract}
We obtain formulas for the spectral zeta function of the Laplacian on symmetric finitely ramified fractals, such as the Sierpiński gasket, and a fractal Laplacian on the interval. These formulas contain a new type of zeta function associated with a polynomial (rational functions also can appear in this context). It is proved that this zeta function has a meromorphic continuation to a half-plane with poles contained in an arithmetic progression. It is shown as an example that the Riemann zeta function is the zeta function of a quadratic polynomial, which is associated with the Laplacian on an interval. The spectral zeta function of the Sierpiński gasket is a product of the zeta function of a polynomial and a geometric part; the poles of the former are canceled by the zeros of the latter. A similar product structure was discovered by M.L. Lapidus for self-similar fractal strings.
\end{abstract}

\section{INTRODUCTION}

In this paper we obtain formulas for the spectral zeta function of the Laplacian on symmetric finitely ramified (or p.c.f., see [20, 21]) fractals, such as the Sierpiński gasket and an interval with a fractal measure. These formulas involve a new type of zeta function associated with a polynomial, and we show that this zeta function has a meromorphic continuation to a strip beyond the half-plane where it is analytic. In the case of the Sierpiński gasket the spectral zeta function has a product structure, with one term which can be described as a "geometric" part, and the other term which is the zeta function of a certain quadratic polynomial. Both of these features, the existence of a meromorphic continuation and a product formula, are reminiscent of the theory of one-dimensional fractal strings first defined and studied by M. Lapidus in 26]. We present basic definitions and results in Section 2 , and the reader can find more details and references in 27, 28, 29, 30, 31, 32. In particular, equations (5.2)-(5.3) in [26] (see also section 1.3 of [27]) contain the product formula

$$
\zeta_{L}(s)=\zeta(s) \zeta_{\mathcal{L}}(s)
$$

where $\zeta(s)$ is the Riemann zeta function, and

$$
\zeta_{\mathcal{L}}(s)=\sum l_{k}^{s}
$$

Received by the editors May 27, 2005 and, in revised form, August 16, 2005.

2000 Mathematics Subject Classification. Primary 28A80, 37F10; Secondary 20H05, 35P20, 37A30, 47A10, 58C40.

Key words and phrases. Spectral zeta function, fractal, rational complex dynamics, Laplacian, fractal string.

This research was supported in part by NSF grants DMS-0071575 and DMS-0505622.

(C)2007 American Mathematical Society Reverts to public domain 28 years from publication 
is the geometric zeta function of a fractal string $\mathcal{L}$, a disjoint collection of intervals of lengths $l_{k}$. The spectral zeta function $\zeta_{L}(s)$ of a normalized Dirichlet Laplacian $L$ on $\mathcal{L}$ is defined as

$$
\zeta_{L}(s)=\sum \lambda_{j}^{-s / 2}
$$

where $\lambda_{j}$ are the nonzero eigenvalues of $L$.

The first step in our work is to define and study a new type of zeta function associated with a polynomial (see Section 3). Suppose that $R(z)$ is a polynomial of degree $N$ such that $R(0)=0, c=R^{\prime}(0)>1$, and the Julia set of $R(z)$ lies in $\mathbb{R}_{+}$. Then we define the zeta function of the polynomial $R(z)$ as

for

$$
\zeta_{R, z_{0}}(s)=\lim _{n \rightarrow \infty} \sum_{z \in R^{-n}\left\{z_{0}\right\}}\left(c^{n} z\right)^{-s / 2}
$$

$$
\operatorname{Re}(s)>d_{R}=\frac{2 \log N}{\log c},
$$

where $R^{-n}\left\{z_{0}\right\}$ means the set of $N^{n}$ preimages of $z_{0}$ under the $n$th composition power of the polynomial $R$. Moreover, we prove that there is an increasing unbounded sequence of positive real numbers $\lambda_{j}$ such that $\zeta_{R, z_{0}}$ can be represented by the Dirichlet series

$$
\zeta_{R, z_{0}}(s)=\sum_{j=1}^{\infty} \lambda_{j}^{-s / 2}
$$

if (1.4) holds. We give examples where the $\lambda_{j}$ are actually eigenvalues of a Laplacian, but it is possible to define $\lambda_{j}$ in terms of $R$ and $z_{0}$ only, without reference to any Laplacian.

Although the definition above make sense under less restrictive conditions on $R(z)$, they are automatically satisfied if the polynomial is associated with a selfsimilar Laplacian (see [38, 33), as will be the case in the applications we consider.

Our main result is that $\zeta_{R, z_{0}}(s)$ has a meromorphic continuation of the form

$$
\zeta_{R, z_{0}}(s)=\frac{\xi_{R}(s)}{1-N c^{-s / 2}}+\psi_{R, z_{0}}(s),
$$

where $\xi_{R}(s)$ is analytic in $\mathbb{C}$ and $\psi_{R, z_{0}}(s)$ is analytic for $\operatorname{Re}(s)>0$. If $\mathcal{J}_{R}$ is totally disconnected, then there exists $\varepsilon>0$ such that $\psi_{R, z_{0}}(s)$ is analytic for $\operatorname{Re}(s)>-\varepsilon$. The set of poles of $\zeta_{R, z_{0}}(s)$ is contained in $\left\{\frac{2 \log N+4 i n \pi}{\log c}: n \in \mathbb{Z}\right\}$, and there always is a pole at $d_{R}$.

Independently of our work this result was recently (after the recent paper was completed) improved in [12 based on the ideas of an earlier paper [18] by Peter Grabner. In particular, it is shown in 12 that $\zeta_{R, z_{0}}$ actually has a meromorphic continuation to the entire complex plane for any polynomial $R$ satisfying the same conditions as in our paper. Moreover, 12] contains a detailed analysis of $\zeta_{R, z_{0}}$ in the case of the Sierpiński gasket. However, an advantage of our method is that it is also applicable in the case when $R$ is a rational function satisfying appropriate assumptions. Therfore it is likely that our results can be extended to the class of fractals considered in 33 . 
One of the reasons this new class of zeta functions is interesting is that the Riemann zeta function $\zeta(s)$ is the zeta function of a quadratic polynomial (Theorem [3). This result is obtained by comparing two representations of the Riemann zeta function: as the Dirichlet series and as the spectral zeta function of the standard Laplacian on an interval. Then the interval can be considered as a self-similar fractal, and the spectrum of the Laplacian can be described in terms of the quadratic polynomial. This construction is not unique in the sense that the Riemann zeta function can be represented as the zeta function of a polynomial of any degree (see Theorem 3 for an example). In Section [5 this construction is generalized for fractal Laplacians on the interval, that is, Laplacians associated with a fractal self-similar measure.

In Section 6 we obtain the following formula for the spectral zeta function of the Laplacian $\Delta_{\mu}$ on the Sierpiński gasket:

$$
\zeta_{\Delta_{\mu}}(s)=\frac{1}{2} \zeta_{R, \frac{3}{4}}(s)\left(\frac{1}{5^{s / 2}-3}+\frac{3}{5^{s / 2}-1}\right)+\frac{1}{2} \zeta_{R, \frac{5}{4}}(s)\left(\frac{3 \cdot 5^{-s / 2}}{5^{s / 2}-3}-\frac{5^{-s / 2}}{5^{s / 2}-1}\right),
$$

where $R(z)=z(5-4 z)$.

Combining (1.6) and (1.7) we obtain that the spectral zeta function of the Sierpiński gasket has a form which resembles the spectral zeta function of a self-similar fractal string (1.1). For example, for the Cantor self-similar fractal string $\mathcal{L}$, which is the complement of the middle third Cantor set in $[0,1]$, we have

$$
\zeta_{L}(s)=\zeta(s) /\left(3^{s}-2\right) .
$$

Thus the spectral zeta function of the Sierpiński gasket has the same structure as the spectral zeta function of a disjoint collection of "fractal intervals". However, this analogy is somewhat superficial because such "fractal intervals" so far have not been constructed or defined. This means that the spectrum has a product structure which does not manifest itself in the structure of the underlying space.

The poles of a spectral zeta function are called the complex spectral dimensions (see [26, 27, 28]). They are 1 and $\left\{\frac{\log 2+2 i n \pi}{\log 3}: n \in \mathbb{Z}\right\}$ for the Cantor self-similar fractal string, and $\left\{\frac{2 i n \pi}{\log 5}, \frac{\log 9+2 i n \pi}{\log 5}: n \in \mathbb{Z}\right\}$ for the Sierpiński gasket; see Figures 2.2 and 6.3. An interesting feature of the product formula (1.7) is that, in the case of the Sierpiński gasket, the poles of the "polynomial" part of $\zeta_{\Delta_{\mu}}(s)$ are canceled by the zeros of the "geometric" part of $\zeta_{\Delta_{\mu}}(s)$. We conjecture that such a cancelation takes place for other symmetric fractals and can be explained by the interplay between geometric and analytic properties of the fractals.

The main results of this paper were announced, without proofs, in [40] with a few typos, which we correct in the present paper.

The author very much appreciates interesting and useful remarks and references by P. Grabner. The author thanks the anonymous referee for many helpful suggestions.

\section{The SPECTRAL ZETA FUnCtion OF A SELF-SIMILAR STRING}

In this expository section we follow the work of Lapidus et al. (see [26, 27, 28, 29, 30, 31, 32 and the references therein).

Definition 2.1. If $A$ is a nonnegative self-adjoint operator with discrete spectrum and positive eigenvalues $\left\{\lambda_{j}\right\}$, counted with their multiplicities, then its spectral 


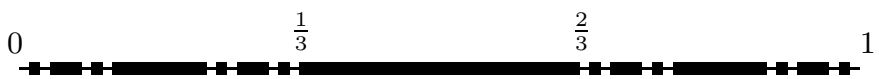

Figure 2.1. Cantor self-similar fractal string

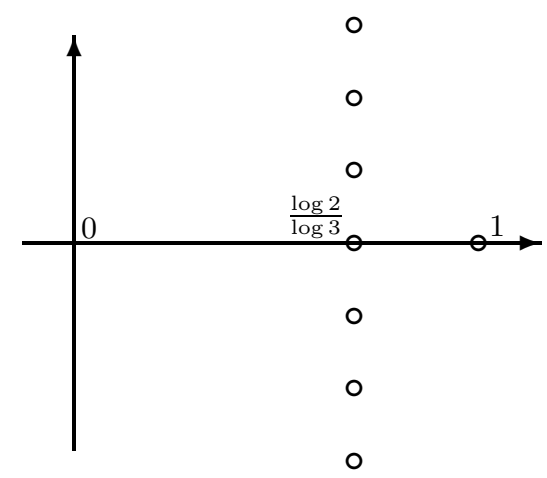

Figure 2.2. Complex spectral dimensions of the Cantor selfsimilar fractal string

zeta function is

$$
\zeta_{A}(s)=\sum \lambda_{j}^{-s / 2}
$$

Note that zero is not included in the sum, even if it is an eigenvalue.

Example 2.2. If $A=-\frac{d^{2}}{d x^{2}}$ is a Neumann or Dirichlet Laplacian on $[0,1]$, then

$$
\zeta_{A}(s)=\sum_{j=1}^{\infty}\left(\frac{j^{2}}{\pi^{2}}\right)^{s / 2}=\pi^{s} \zeta(s),
$$

where $\zeta(s)$ is the Riemann zeta function. The series converges for $\operatorname{Re}(s)>1$ and has a meromorphic continuation to $\mathbb{C}$ with one pole at $s=1$.

Definition 2.3. The poles of $\zeta_{A}(s)$ are called the complex spectral dimensions of $A$.

Definition 2.4. If $\mathcal{L}$ is a fractal string, that is, a disjoint collection of intervals of lengths $l_{k}$, then its geometric zeta function is

$$
\zeta_{\mathcal{L}}(s)=\sum l_{k}^{s} \text {. }
$$

The next theorem is due to M.L. Lapidus 26, 27, 28, 29, 30, 31, 32,

Theorem. If $A=-\frac{d^{2}}{d x^{2}}$ is a Neumann or Dirichlet Laplacian on $\mathcal{L}$, then

$$
\zeta_{A}(s)=\pi^{s} \zeta(s) \zeta_{\mathcal{L}}(s) \text {. }
$$

Example 2.5 (a self-similar fractal string). Let $r>1$ and $l_{j}=c r^{-k}$ for $N^{k}$ intervals, where $N$ is a positive integer, $k=0,1,2, \ldots$. Then

$$
\zeta_{\mathcal{L}}(s)=\frac{c^{s}}{1-N r^{-s}}, \quad \zeta_{A}(s)=\zeta(s) \frac{c^{s} \pi^{s}}{1-N r^{-s}} .
$$


If $N<r$, then the total length of $\mathcal{L}$ is finite and the spectral dimension is 1 ; otherwise it is $\frac{\log N}{\log r}$. The complex spectral dimensions are 1 and $\left\{\frac{\log N+2 i n \pi}{\log r}: n \in\right.$ $\mathbb{Z}\}$.

For example, for the Cantor self-similar fractal string, that is, the complement of the middle third Cantor set in $[0,1]$, we have $c=\frac{1}{3}, r=3, N=2$.

\section{The Zeta FunCtion of A POLYNOMial}

We suppose that $R(z)$ is a polynomial of degree $N$ such that $R(0)=0$ and $c=R^{\prime}(0)>1$. We also assume that the Julia set $\mathcal{J}_{R}$ of $R(z)$ satisfies $\mathcal{J}_{R} \subset \mathbb{R}_{+}$. These assumptions imply immediately that the coefficients of $R$ are real. Since zero is a repulsive fixed point, its preimages are dense in $J_{R}$ by the classical paper [10. Thus, $\mathcal{J}_{R} \subset \mathbb{R}_{+}$if and only if there is a real interval $[0, a] \subset \mathbb{R}_{+}$such that $R^{-1}[0, a] \subset[0, a]$.

By [10, the Julia set in our situation is an interval or a Cantor set of Lebesgue measure zero. We denote the convex hull of $\mathcal{J}_{R}$ by $I$, which is an interval. By the same paper, if $\mathcal{J}_{R}$ is an interval, then $R^{-n}(I)=I$. Otherwise $R^{-n}(I) \varsubsetneqq I$ consists of $N^{n}$ disjoint intervals. In both cases $R^{-n-1}(I) \subset R^{-n}(I)$ and

$$
J_{R}=\bigcap_{n=1}^{\infty} R^{-n}(I),
$$

where $R^{-n}(\cdot)$ denotes the preimage under the $n$th composition (iteration) power of the polynomial $R(z)$. Also one has a unique decomposition $I=\bigcup_{j=1}^{N} I_{j}$, where $I_{j}$ are intervals with disjoint interior, and $R(z)$ is monotone on each $I_{j}$.

In what follows we denote $I_{0}=\bigcap_{j=1}^{N} R\left(I_{j}\right)$. Then we have $R^{-1}\left(I_{0}\right) \subseteq I \subseteq I_{0}$.

Definition 3.1. The zeta function of the polynomial $R(z)$ is

$$
\zeta_{R, z_{0}}(s)=\lim _{n \rightarrow \infty} \sum_{z \in R^{-n}\left\{z_{0}\right\} \backslash 0}\left(c^{n} z\right)^{-s / 2},
$$

where $z_{0} \in I_{0}$, and $\operatorname{Re}(s)>d_{R}$. Here we define

$$
d_{R}=\frac{2 \log N}{\log c} .
$$

Theorem 1. For $\operatorname{Re}(s)>d_{R}$ the limit (3.1) exists and is an analytic function of s. Moreover, there is a nondecreasing unbounded sequence of positive real numbers $\lambda_{j}$ such that

where $\lambda_{j}$ are defined in terms of $R$ and $z_{0}^{j=1}$

$$
\zeta_{R, z_{0}}(s)=\sum_{j=1}^{\infty} \lambda_{j}^{-s / 2}
$$

Proof. By monotonicity, the restriction of the polynomial $R(z)$ to each $I_{j}$ has an inverse, which we denote by $R_{j}^{-1}$. We assume that the intervals $I_{j}$ are numbered consecutively starting from the origin. In particular, $R_{1}^{-n}(\cdot)$ is the $n$th composition power of the branch of the inverse function of $R(z)$ that passes through the origin.

We denote by $\mathcal{J}_{+}$the part of $\mathcal{J}_{R}$ that is not in $R_{1}^{-1}(I)$, that is,

$$
\mathcal{J}_{+}=\mathcal{J}_{R} \cap\left(\bigcup_{k=2}^{N} R_{k}^{-1}(I)\right)=\bigcup_{k=2}^{N} R_{k}^{-1}\left(\mathcal{J}_{R}\right) .
$$


We denote by $\mathcal{R}$ the limit

$$
\mathcal{R}(z)=\lim _{n \rightarrow \infty} c^{n} R_{1}^{-n}(z) .
$$

Since $R^{\prime}(0)=c$, this limit exists for any $z \in I_{0}$ and satisfies $\mathcal{R}(z)=c \mathcal{R}\left(R_{1}^{-1}(z)\right)$ and is an increasing continuous function. The function $\mathcal{R}$ was first defined and studied by P. Fatou in 14. Its analytic properties are well studied in complex dynamics, but we need only the elementary facts that the limit (3.4) exists and is an increasing continuous function for real $z \in I_{0}$. To show this, notice that $R^{\prime}(z)>0$ and $R^{\prime \prime}(z)<0$ in the interior of $I_{1}$ by [10. Then it is easy to see that $z_{n}=R_{1}^{-n}(z)$ converges to zero exponentially fast. Hence

$$
c z_{n+1}=c R_{1}^{-1}\left(z_{n}\right)=z_{n}+O\left(z_{n}^{2}\right)_{n \rightarrow \infty}=z_{n}\left(1+O\left(z_{n}\right)_{n \rightarrow \infty}\right),
$$

which proves (3.4).

Let $W_{n}$ be the set of sequences (words) $w=k_{1} \ldots k_{n}$ of length $n$, where $k_{i} \in$ $\{1, \ldots, N\}$. For a word $w=k_{1} \ldots k_{n} \in W_{n}$ we write $R_{w}^{-n}=R_{k_{1}}^{-1} \ldots R_{k_{n}}^{-1}$. We use the convention that $W_{0}=\{\varnothing\}$ and $R_{w}^{0}(z)=z$ for $w=\varnothing \in W_{0}$.

Let $W_{n}^{+}$be a subset of $W_{n}$ of words that do not start with 1 . Then we have

$$
\begin{aligned}
\zeta_{R, z_{0}}(s) & =\lim _{n \rightarrow \infty} \sum_{z \in R^{-n}\left\{z_{0}\right\}}\left(c^{n} z\right)^{-s / 2} \\
& =\lim _{n \rightarrow \infty} \sum_{m=0}^{n} c^{-s m / 2} \sum_{w \in W_{m}^{+}}\left(c^{n-m} R_{1}^{-n+m} R_{w}^{-m}\left(z_{0}\right)\right)^{-s / 2} \\
& =\sum_{m=0}^{\infty} c^{-s m / 2} \sum_{w \in W_{m}^{+}}\left(\mathcal{R} R_{w}^{-m}\left(z_{0}\right)\right)^{-s / 2}
\end{aligned}
$$

for $\operatorname{Re}(s)>d_{R}$.

Here and in what follows, in order to simplify notation, we use the convention that $z^{-s / 2}=0$ for all real $s$ if $z=0$.

The last expression in (3.5) can be rewritten as

$$
\zeta_{R, z_{0}}(s)=\sum_{m=0}^{\infty} \sum_{w \in W_{m}^{+}}\left(c^{m} \mathcal{R}\left(R_{w}^{-m}\left(z_{0}\right)\right)\right)^{-s / 2}=\sum_{j=1}^{\infty} \lambda_{j}^{-s / 2},
$$

where the nondecreasing unbounded sequence of positive real numbers $\left\{\lambda_{j}\right\}_{j=1}^{\infty}$ is obtained by reordering the countable locally finite set of positive real numbers $\bigcup_{m=0}^{\infty} \bigcup_{w \in W_{m}^{+}}\left\{c^{m} \mathcal{R}\left(R_{w}^{-m}\left(z_{0}\right)\right)\right\} \backslash\{0\}$, taking the multiplicities into account.

Theorem 2. The zeta function $\zeta_{R, z_{0}}(s)$ of the polynomial $R(z)$ has a meromorphic continuation of the form

$$
\zeta_{R, z_{0}}(s)=\frac{\xi_{R}(s)}{1-N c^{-s / 2}}+\psi_{R, z_{0}}(s),
$$

where $\xi_{R}(s)$ is analytic in $\mathbb{C}$ and $\psi_{R, z_{0}}(s)$ is analytic for $\operatorname{Re}(s)>0$ and is defined by (3.7). The set of poles of $\zeta_{R, z_{0}}(s)$ is contained in

$$
\left\{d_{R}+\frac{4 i n \pi}{\log c}: n \in \mathbb{Z}\right\}
$$

and there always is a pole at $d_{R}$. 
If $\mathcal{J}_{R}$ is totally disconnected, then there exists $\varepsilon>0$ such that $\psi_{R, z_{0}}(s)$ is analytic for $\operatorname{Re}(s)>-\varepsilon$. If, in addition, $r=\max _{z \in \mathcal{J}_{R}}\left|R^{\prime}(z)\right|^{-1}<1$, then we can take

$$
\varepsilon=-\frac{2 \log r}{\log c} .
$$

Independently of our work this result was recently improved in [12. In particular, it is shown in 12] that $\zeta_{R, z_{0}}$ has a meromorphic continuation to the entire complex plane for any polynomial $R$ satisfying the same conditions as in our paper. However, an advantage of our method is that it is also applicable in the case when $R$ is a rational function satisfying appropriate assumptions. Therefore it is likely that our results can be extended to the class of fractals considered in [33.

Proof of Theorem 2. To the last expression in (3.5) we add and subtract

$$
\frac{\xi_{R}(s)}{1-N c^{-s / 2}}
$$

where

$$
\xi_{R}(s)=\int_{\mathcal{J}_{+}}(\mathcal{R}(z))^{-s / 2} d \kappa(z) .
$$

Here we use the fact (see, for instance, $\left[5,7,8,10\right.$, ) that on $\mathcal{J}_{R}$ there is a unique balanced invariant probability measure $\kappa$ which satisfies

for any sequence $j_{1}, \ldots, j_{m}$.

$$
\kappa\left(R_{j_{1}}^{-1} \cdots R_{j_{m}}^{-1}(I)\right)=\frac{1}{N^{m}}
$$

Note that $\xi_{R}(s)$ is an analytic function since $\mathcal{J}_{+}$is separated from zero, and that $\frac{1}{1-N c^{-s / 2}}=\sum_{m=0}^{\infty} N^{m} c^{-s m / 2}$. Then we only have to show that

$$
\begin{aligned}
& \sum_{m=0}^{\infty} c^{-s m / 2}\left(\sum_{w \in W_{m}^{+}}\left(\mathcal{R} R_{w}^{-m}\left(z_{0}\right)\right)^{-s / 2}-N^{m} \int_{\mathcal{J}_{+}}(\mathcal{R}(z))^{-s / 2} d \kappa(z)\right) \\
& =\sum_{m=0}^{\infty} N^{m} c^{-s m / 2} \sum_{w \in W_{m}^{+}} \int_{R_{w}^{-m}\left\{\mathcal{J}_{+}\right\}}\left[\left(\mathcal{R} R_{w}^{-m}\left(z_{0}\right)\right)^{-s / 2}-(\mathcal{R}(z))^{-s / 2}\right] d \kappa(z)
\end{aligned}
$$

is analytic when $\operatorname{Re}(s)>0$.

Thus we have to show that the series converges uniformly when $s$ is in a compact subset. To this end we can estimate the absolute value of the expression in square brackets by

$$
M \max _{z \in R_{w}^{-m}\left\{\mathcal{J}_{+}\right\}}\left|z-R_{w}^{-m}\left(z_{0}\right)\right| \leqslant M \lambda\left(R_{w}^{-m}\left\{I_{0}\right\}\right),
$$

where $\lambda$ is the Lebesgue measure on $\mathbb{R}$,

$$
M=\max _{z \in I_{+}}\left|\frac{d}{d z}(\mathcal{R}(z))^{-s / 2}\right|
$$

and $I_{+}=\bigcup_{w \in W_{1}^{+}} R_{w}^{-1}\left\{I_{0}\right\}$.

Then, since $\kappa\left(R_{w}^{-m}\{\mathcal{J}\}\right)=N^{-m}$,

$$
\sum_{w \in W_{m}^{+}} \int_{R_{w}^{-m}\left\{\mathcal{J}_{+}\right\}}\left|\left(\mathcal{R} R_{w}^{-m}\left(z_{0}\right)\right)^{-s / 2}-(\mathcal{R}(z))^{-s / 2}\right| d \kappa(z) \leqslant M N^{-m} \lambda\left(R^{-m+1}(I)\right) .
$$


In this estimate we have to use the exponent $-m+1$ instead of $m$, because we assumed that $z_{0} \in I_{0}$, not necessarily $z_{0} \in I$.

Thus we have the required convergence for $\operatorname{Re}(s)>0$.

If $\mathcal{J}_{R}$ is totally disconnected, then by pages 122-124 in [10, there is $r<1$ such that

$$
\lambda\left(R^{-n}(I)\right)<\mathrm{const} \cdot r^{n} .
$$

This estimate gives the required convergence for $\operatorname{Re}(s)>-\varepsilon=\frac{2 \log r}{\log c}$. Finally, note that $r \leqslant \max _{z \in \mathcal{J}_{R}}\left|R^{\prime}(z)\right|^{-1}$.

In the applications of this theorem considered in this paper, the assumptions on the polynomial $R(z)$ given above are always satisfied, because the Julia set of $R(z)$ is contained in the spectrum of a nonnegative self-adjoint operator (see [33]). Moreover,

$$
\zeta_{R, z_{0}}(s)=\sum \lambda_{j}^{-s / 2}
$$

where $\left\{\lambda_{j}\right\}$ are eigenvalues of a Laplacian, and so the spectral zeta function has a representation as a zeta function of a polynomial. In these cases the parameter $z_{0}$ usually depends on the boundary conditions. However, even if the polynomial $R(z)$ is not related to any Laplacian, the representation (3.8) still holds with $\left\{\lambda_{j}\right\}$ defined in terms of the limit points of $\bigcup_{n>0} c^{n} R^{-n}\left(z_{0}\right)$ as in Theorem 1 .

Theorem 3. The Riemann zeta function $\zeta(s)$ has a representation

$$
\zeta(s)=\frac{1}{2} C^{s} \zeta_{R, 0}(s)
$$

where $C=\sqrt{2} \pi$ and $\zeta_{R, 0}(s)$ is the zeta function of the polynomial $R(z)=2 z(2-z)$.

Proof. For the Neumann or Dirichlet Laplacian $\Delta$ on $I=[0,1]$ we have

$$
\zeta(s)=\sum_{j=1}^{\infty} j^{-s}=\pi^{s} \sum_{j=1}^{\infty}\left(\pi^{2} j^{2}\right)^{-s / 2}=\pi^{s} \zeta_{\Delta}(s) .
$$

Let

$$
\Delta_{n} f\left(\frac{k}{2^{n}}\right)=f\left(\frac{k}{2^{n}}\right)-\frac{1}{2} f\left(\frac{k-1}{2^{n}}\right)-\frac{1}{2} f\left(\frac{k+1}{2^{n}}\right)
$$

be the probabilistic Laplacian on $\left\{0, \ldots, \frac{k}{2^{n}}, \frac{k+1}{2^{n}}, \ldots, 1\right\}$, which is the generator of the simple nearest neighbor random walk. Then

$$
\Delta f(x)=-f^{\prime \prime}(x)=2 \lim _{n \rightarrow \infty} 4^{n} \Delta_{n} f(x)
$$

for any twice continuously differentiable function $f$. The theorem then follows from the definition of $\zeta_{R, 0}(s)$ and Lemma 3.2 since

$$
\zeta_{\Delta}(s)=\frac{1}{2} 2^{-s / 2} 4^{s / 2} \zeta_{R, 0}(s)=\frac{1}{2} 2^{s / 2} \zeta_{R, 0}(s) .
$$

Note that the preimages of zero are $\{0,2\}=\sigma\left(\Delta_{0}\right)$, and so the spectrum of $\Delta_{n}$ as a set coincides with $R^{-n-1}(0)$, which explains the extra factor $4^{s / 2}$ above. Also note that 1 is the preimage of 2 of multiplicity two, but 1 is a simple eigenvalue of $\Delta_{n}$, which explains the extra factor $\frac{1}{2}$ above.

Lemma 3.2. If $z \neq 1$, then $R(z)=2 z(2-z) \in \sigma\left(\Delta_{n}\right)$ if and only if $z \in \sigma\left(\Delta_{n+1}\right)$, with the same multiplicities. Moreover, the Neumann discrete Laplacians have simple spectrum with $\sigma\left(\Delta_{0}\right)=\{0,2\}$ and $1 \in \sigma\left(\Delta_{n}\right)$ for all $n>0$. 
Proof. This lemma can be proved by an elementary computation; see [33, Example 2.4 and Theorem 5.8].

\section{Mellin transform}

In this expository section we contrast the zeta function of a polynomial with the Mellin transform of an invariant measure related to the same polynomial. We do not use the correspondence between these objects in our work, but the reader can find some related formulas in 12,18 .

Definition 4.1. If $\kappa$ is the unique balanced invariant measure on the Julia set $\mathcal{J}_{R}$ of $R(z)$ (see Figure 4.1), then its Mellin transform is

$$
\mathcal{M}_{R}(s)=\lim _{n \rightarrow \infty} N^{-n} \sum_{z \in R^{-n}\left\{z_{0}\right\}} z^{-s / 2}=\int_{\mathcal{J}_{R}} z^{-s / 2} d \kappa(z) .
$$

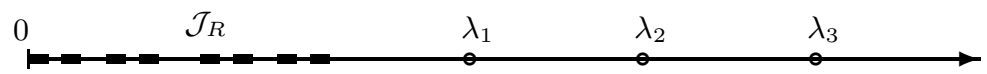

Figure 4.1. A pictorial illustration of the Julia set $\mathcal{J}_{R}$ of $R(z)$ and the sequence $\left\{\lambda_{j}\right\}$ in Theorem 1

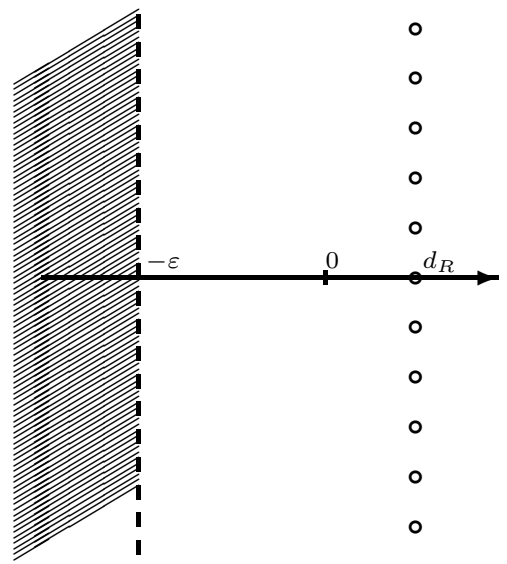

\begin{tabular}{|c|c|c|c|}
\hline & $\circ$ & $\circ$ & $\circ$ \\
\hline & $\circ$ & $\circ$ & $\circ$ \\
\hline & $\circ$ & $\circ$ & ० \\
\hline & $\circ$ & $\circ$ & $\circ$ \\
\hline & ○ & ○ & $\circ$ \\
\hline+ & $d_{R}$ & $d_{R}+1$ & $d_{R}+2$ \\
\hline & ○ & ○ & ० \\
\hline & ○ & ० & ० \\
\hline & ○ & ○ & $\circ$ \\
\hline & ○ & ० & $\circ$ \\
\hline & 0 & $\circ$ & ० \\
\hline
\end{tabular}

Figure 4.2. A pictorial illustration of the poles of the zeta function of a polynomial in Theorem 2 (left), and of the poles of its Mellin transform (right)

Theorem 4 ([7]). The Mellin transform $\mathcal{M}_{R}$ converges for $\operatorname{Re}(s)<d_{R}$ and has a meromorphic continuation to $\mathbb{C}$ with the set of poles contained in

$$
\left\{\frac{2 \log N+4 i n \pi}{\log c}+m: n \in \mathbb{Z}, m \in \mathbb{N}\right\} .
$$

Every real pole has a positive residue and thus is not degenerate.

Note that by the Theorem of Bessis, Geronimo and Moussa the Mellin transform has infinitely many nondegenerate positive real poles, while by Theorem 2 the zeta function of a polynomial has only one positive real pole (see Figure 4.2). 


\section{Fractal LAPLACIAN ON AN INTERVAL AND ITS ZETA FUNCTION}

We begin with defining the usual energy form of the unit interval by introducing a self-similar structure. In this way the interval can be considered as a p.c.f. fractal (see [2, 9, 20, 21]). In particular, in these papers the reader can find proofs of the propositions given below, as well as the general theory of Dirichlet forms on fractals and further references.

We use three contractions because it will allow us to easily modify the construction in order to obtain a fractal Laplacian on the unit interval. One can make the same construction using any number of contractions. Note that in Theorem 3 we essentially used a self-similar structure on the unit interval with two contractions.

Remark 5.1. We emphasize that in this and the next section we consider two different iteration function systems which are not to be confused. We start with an iteration function system of linear contractions to define a self-similar set, which is the set where the Laplacian is defined (and the state space of the corresponding diffusion process). This self-similar set is often a fractal, but in this section it is an interval with a self-similar structure. Then, based on the "spectral decimation" or, more appropriately called, "spectral self-similarity" (see [33]), we describe the spectrum of the Laplacian using inverse iterations of a polynomial (a rational function in the more general situation of 33 ). To this latter iteration function system we apply the results of Section 3 . by

We define three contractions $F_{1}, F_{2}, F_{3}: \mathbb{R}^{1} \rightarrow \mathbb{R}^{1}$ with fixed points $x_{j}=0, \frac{1}{2}, 1$

$$
F_{j}(x)=\frac{1}{3} x+\frac{2}{3} x_{j}
$$

Then the interval $I=[0,1]$ is a unique compact set such that

$$
I=\bigcup_{j=1,2,3} F_{j}(I) .
$$

The discrete approximations to $I$ are defined inductively by

$$
V_{n}=\bigcup_{j=1,2,3} F_{j}\left(V_{n-1}\right)=\left\{\frac{k}{3^{n}}\right\}_{k=0}^{3^{n}},
$$

where $V_{0}=\partial I=\{0,1\}$ is the boundary of $I$. For $x, y \in V_{n}$ we write $y \sim x$ if $|x-y|=3^{-n}$.

Definition 5.2. The discrete Dirichlet (energy) form on $V_{n}$ is

$$
\mathcal{E}_{n}(f, f)=3^{n} \sum(f(y)-f(x))^{2},
$$

where the sum is taken over all unordered pairs $x, y \in V_{n}$ such that $y \sim x$. The Dirichlet (energy) form on $I$ is

$$
\mathcal{E}(f, f)=\lim _{n \rightarrow \infty} \mathcal{E}_{n}(f, f)
$$

if this limit exists and is finite. By the next proposition this limit is increasing and so it always exists as a nonnegative real number or $+\infty$.

A function $h$ is harmonic if it minimizes the energy given the boundary values.

Proposition 5.3. We have that $\mathcal{E}_{n+1}(f, f) \geqslant \mathcal{E}_{n}(f, f)$ for any function $f$, and

$$
\mathcal{E}_{n+1}(h, h)=\mathcal{E}_{n}(h, h)=\mathcal{E}(h, h)
$$


for a harmonic $h$. A function $h$ is harmonic if and only if it is linear. If $f$ is continuously differentiable, then

$$
\mathcal{E}(f, f)=\int_{0}^{1}\left|f^{\prime}(x)\right|^{2} d x
$$

The Dirichlet (energy) form $\mathcal{E}$ on I is self-similar in the sense that

$$
\mathcal{E}(f, f)=3 \sum_{j=1,2,3} \mathcal{E}\left(f \circ F_{j}, f \circ F_{j}\right) .
$$

Definition 5.4. The discrete Laplacians on $V_{n}$ are

$$
\Delta_{n} f(x)=\frac{1}{2} \sum_{\substack{y \in V_{n} \\ y \sim x}}(f(y)-f(x)), \quad x \in V_{n} \backslash V_{0},
$$

and the (renormalized) Laplacian on $I$ is

$$
\Delta f(x)=\lim _{n \rightarrow \infty} 9^{n} \Delta_{n} f(x)=\frac{1}{2} f^{\prime \prime}(x)
$$

for any twice differentiable function. Obviously, for any twice differentiable function this Laplacian satisfies the Gauss-Green (integration by parts) formula

$$
\mathcal{E}(f, f)=-2 \int_{0}^{1} f \Delta f d x+\left.f f^{\prime}\right|_{0} ^{1}
$$

and has the spectral asymptotics

$$
\lim _{\lambda \rightarrow \infty} \frac{\rho(\lambda)}{\lambda^{d_{s} / 2}}=\frac{1}{\pi},
$$

where $\rho(\lambda)$ is the eigenvalue counting function $\rho(\lambda)=\#\left\{j: \lambda_{j}<\lambda\right\}$ of the Dirichlet or Neumann Laplacian $\Delta$ and $d_{s}=1$ is its spectral dimension.

In what follows we modify this construction to obtain a one-parameter family of fractal Laplacians on the unit interval. We introduce a parameter $0<p<1$ and define $q=1-p$, which later will be shown to have a meaning of transitional probabilities of a random walk. Then we define contraction factors (or "resistance weights")

$$
r_{1}=r_{3}=\frac{p+p q}{2+p q} \quad \text { and } \quad r_{2}=\frac{2 q-p q}{2+p q},
$$

and "measure weights"

$$
m_{1}=m_{3}=\frac{q}{1+q} \quad \text { and } \quad m_{2}=\frac{p}{1+q} .
$$

Note that, up to a constant factor, the resistance weights are reciprocals of the measure weights, and

$$
m_{1}+m_{2}+m_{3}=r_{1}+r_{2}+r_{3}=1 .
$$

By their definition, constants, resistance weights $r_{i}$ and measure weights $m_{i}$ depend on the choice of $p$.

We define three contractions $F_{p, 1}, F_{p, 2}, F_{p, 3}: \mathbb{R}^{1} \rightarrow \mathbb{R}^{1}$ with fixed points $x_{j}=$ $0, \frac{1}{2}, 1$ by

$$
F_{p, j}(x)=r_{j} x+\left(1-r_{j}\right) x_{j} .
$$


Then the interval $I=[0,1]$ is a unique compact set such that

$$
I=\bigcup_{j=1,2,3} F_{p, j}(I)
$$

The discrete approximations to $I$ are defined inductively by

$$
V_{p, n}=\bigcup_{j=1,2,3} F_{p, j}\left(V_{p, n-1}\right)
$$

where, as before, $V_{0}=\partial I=\{0,1\}$ is the boundary of $I$.

Definition 5.5. The discrete Dirichlet (energy) form on $V_{n}$ is defined inductively by

$$
\mathcal{E}_{p, n}(f, f)=\sum_{j=1,2,3} \frac{1}{r_{j}} \mathcal{E}_{p, n-1}\left(f \circ F_{j}\right)
$$

with $\mathcal{E}_{0}(f, f)=(f(1)-f(0))^{2}$ as before. The Dirichlet (energy) form on $I$ is

$$
\mathcal{E}_{p}(f, f)=\lim _{n \rightarrow \infty} \mathcal{E}_{p, n}(f, f)
$$

if this limit exist and is finite. Note that this is the same definition as the Dirichlet form defined before if and only if $p=\frac{1}{2}$. By the next proposition this limit is increasing, and so it always exists as a nonnegative real number or $+\infty$.

The next two propositions follow, for example, from the general results in [2, 9 , 20, 21. Again, a function $h$ is harmonic if it minimizes the energy given the three boundary values.

Proposition 5.6. We have that $\mathcal{E}_{p, n+1}(f, f) \geqslant \mathcal{E}_{p, n}(f, f)$ for any function $f$, and

$$
\mathcal{E}_{p, n+1}(h, h)=\mathcal{E}_{p, n}(h, h)=\mathcal{E}_{p}(h, h)
$$

for a harmonic $h$.

Proposition 5.7. The Dirichlet (energy) form $\mathcal{E}_{p}$ on $I$ is local and regular and is self-similar in the sense that

$$
\mathcal{E}_{p}(f, f)=\sum_{j=1,2,3} \frac{1}{r_{j}} \mathcal{E}_{p}\left(f \circ F_{p, j}, f \circ F_{p, j}\right) .
$$

The domains of $\mathcal{E}_{p}$ and of the corresponding Laplacian $\Delta_{\mu}$ are contained and dense in the space of continuous functions.

There exists a $\mu$-Laplacian $\Delta_{\mu}$, that is, a densely defined self-adjoint operator that satisfies the following Gauss-Green (integration by parts) formula:

$$
\mathcal{E}_{p}(f, f)=-C \int_{0}^{1} f \Delta_{\mu} f d \mu+\left.f f^{\prime}\right|_{0} ^{1}
$$

where $\mu$ is a unique probability self-similar measure with weights $m_{1}, m_{2}, m_{3}$, that is,

$$
\mu=\sum_{j=1,2,3} m_{j} \mu \circ F_{p, j}
$$

Also

$$
\Delta_{\mu} f(x)=\lim _{n \rightarrow \infty}\left(1+\frac{2}{p q}\right)^{n} \Delta_{p, n} f(x)
$$




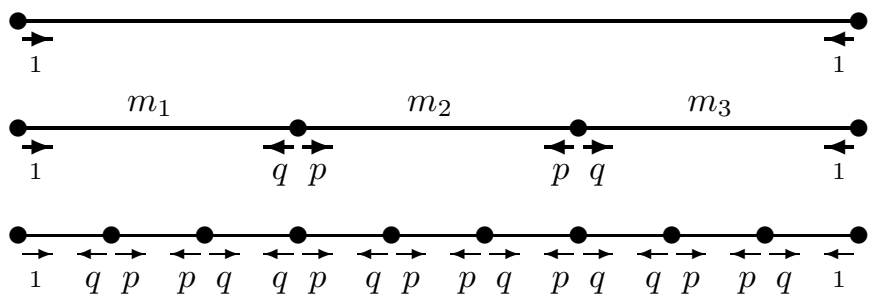

FiguRE 5.1. Random walks corresponding to the discrete Laplacians $\Delta_{p, n}$

where the discrete Laplacians

$$
\Delta_{p, n} f\left(y_{k}\right)=\left\{\begin{array}{l}
p f\left(y_{k-1}\right)+q f\left(y_{k+1}\right)-f\left(y_{k}\right) \\
q f\left(y_{k-1}\right)+p f\left(y_{k+1}\right)-f\left(y_{k}\right)
\end{array}\right. \text { or }
$$

are defined as the generators of the nearest neighbor random walks on $V_{p, n}$ with transitional probabilities $p$ and $q$ assigned according to the weights of the corresponding intervals.

Note that by definition $p=\frac{m_{2}}{m_{1}+m_{2}}, q=\frac{m_{1}}{m_{1}+m_{2}}$. The transitional probabilities $p$ and $q$ can be assigned inductively as shown in Figure 5.1.

Note that the previous construction of the standard Laplacian and the Dirichlet form on $I=[0,1]$ corresponds to $p=\frac{1}{2}$. If $p \neq \frac{1}{2}$, then we can make a change of variable on the unit interval $I$ so that either the Dirichlet form becomes the standard one, or the measure becomes the Lebesgue measure, but not both. Thus for different values of $p$ the $\mu$-Laplacians are different even up to a change of variable.

By the result of Kigami and Lapidus [22, the Dirichlet or Neumann Laplacian $\Delta_{\mu}$ has the spectral asymptotics

$$
0<\liminf _{\lambda \rightarrow \infty} \frac{\rho_{p}(\lambda)}{\lambda^{d_{s} / 2}} \leqslant \limsup _{\lambda \rightarrow \infty} \frac{\rho_{p}(\lambda)}{\lambda^{d_{s} / 2}}<\infty
$$

where $\rho_{p}(\lambda)$ is the eigenvalue counting function of $\Delta_{\mu}$, and its spectral dimension is

$$
d_{s}=\frac{\log 9}{\log \left(1+\frac{2}{p q}\right)} \leqslant 1
$$

where the inequality is strict if and only if $p \neq q$.

In what follows we denote

$$
R_{p}(z)=z\left(z^{2}+3 z+2+p q\right) /(p q) .
$$

We use the notation $\sigma(A)$ for the spectrum of a linear operator $A$.

Lemma 5.8. If $z \neq-1 \pm p$, then $R(z) \in \sigma\left(\Delta_{p, n}\right)$ if and only if $z \in \sigma\left(\Delta_{p, n+1}\right)$, with the same multiplicities. Moreover, the Neumann discrete Laplacians have simple spectrum with $\sigma\left(\Delta_{0}\right)=\{0,-2\}$ and

$$
\sigma\left(\Delta_{p, n}\right)=\{0,-2\} \bigcup_{m=0}^{n-1} R_{p}^{-m}\{-1 \pm q\}
$$


for all $n>0$. In particular, for all $n>0$ we have $0,-1 \pm q,-2 \in \sigma\left(\Delta_{p, n}\right)$. Also, for all $n>0$ we have $-1 \pm p \in \sigma\left(\Delta_{p, n}\right)$ if and only if $p=q$.

Proof. In this case, according to [39, Lemma 3.4], [33, (3.2)] we have that $R_{p}(z)=$ $\frac{\varphi_{1}(z)}{\varphi_{0}(z)}$, where $\varphi_{0}$ and $\varphi_{1}$ solve the matrix equation

$$
S-z I_{0}-\bar{X}\left(Q-z I_{1}\right)^{-1} X=\varphi_{0}(z) H_{0}-\varphi_{1}(z) I_{0}
$$

with $S=\bar{X}=X=I_{0}=I_{1}$, the $2 \times 2$ identity matrices,

$$
Q=\left(\begin{array}{rr}
-1 & p \\
p & -1
\end{array}\right)
$$

and

$$
H_{0}=\left(\begin{array}{rr}
-1 & 1 \\
1 & -1
\end{array}\right)
$$

Solving this we obtain

$$
\varphi_{0}(z)=\frac{p q}{z^{2}+2 z+1-p^{2}}
$$

and

$$
\varphi_{1}(z)=\frac{z\left(z^{2}+3 z+2+p q\right)}{z^{2}+2 z+1-p^{2}} .
$$

Then we use the abstract spectral self-similarity result (see [39, 33]) that the spectrum of $\Delta_{p, n+1}$ can be obtain from the spectrum of $\Delta_{p, n}$ by the inverse of $R(z)$. Note that 0 and 2 are fixed points of $R(z)$. The preimages of 0 are $0,-1-p$ and $-1-q$. The preimages of 2 are $2,-1+p$ and $-1+q$. If $p \neq q$, then $-1 \pm p$ are not eigenvalues because they are poles of $\varphi_{0}(z)$ (see [33, 39]).

Theorem 5. If $p \neq \frac{1}{2}$, then $d_{s}=d_{R}<1$ and the spectral zeta function of $\Delta_{\mu}$ is

$$
\zeta_{\Delta_{\mu}}(s)=\frac{c^{s}}{1-c^{-s / 2}}\left(\zeta_{R, z_{1}}(s)+\zeta_{R, z_{2}}(s)\right)
$$

where $c=1+\frac{2}{p q}$ and $z_{1}, z_{2}=1 \pm q$. Moreover, $\zeta_{\Delta_{\mu}}(s)$ has a meromorphic continuation to $\operatorname{Re}(s)>-\varepsilon$ for some $\varepsilon_{p}>0$, and $\liminf _{p \rightarrow 0} \varepsilon_{p} \geqslant 2, \liminf _{p \rightarrow 1} \varepsilon_{p} \geqslant 2$.

Proof. This follows from Lemma 5.8, Theorem 2 and the definition of the zeta function of a polynomial. The last statement follows from the fact that as $p \rightarrow 0$ or $p \rightarrow 1$ we have $\lim \min _{z \in \mathcal{J}_{R}}\left|R_{p}^{\prime}(z)\right|=\infty$.

Theorem 6. The Riemann zeta function $\zeta(s)$ has a representation

$$
\zeta(s)=\frac{1}{2} C^{s} \zeta_{R, 0}(s)
$$

where $C=\frac{3 \pi}{\sqrt{2}}$ and $\zeta_{R, 0}(s)$ is the zeta function of the polynomial

$$
R(z)=z\left(4 z^{2}-12 z+9\right) .
$$

Proof. The proof follows from Lemma 5.8, Theorem 2 and the definition of the zeta function of a polynomial, analogously to the proof of Theorem 3 , and of Theorem 5 with $p=q$. 


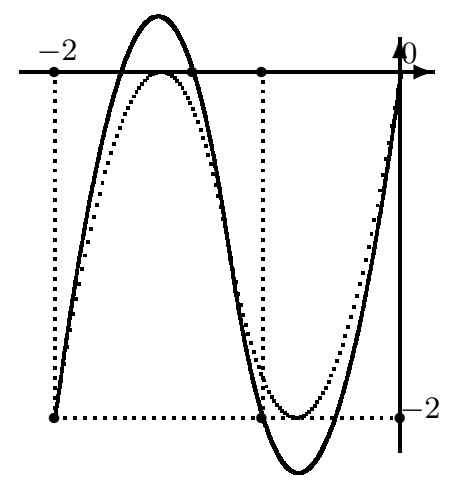

FIGURE 5.2. Sketch of the cubic polynomial $R_{p}(z)$ associated with the fractal Laplacians on the interval

Remark 5.9. In Figure 5.2 we give a sketch that describes the complex dynamics of the family of cubic polynomials associated with the fractal Laplacians on the interval. Note that the Julia set is real, and so what we sketch is actually the real dynamics of these polynomials. The curved dotted line corresponds to the case when $p=\frac{1}{2}$ and the Julia set of $R(z)=R_{\frac{1}{2}}(z)$ is the interval $[-2,0]$. For any other value of $p(0<p<1)$, the graph of the polynomial $R_{p}(z)$ behaves like the solid curved line shown. It is easy to see that then the Julia set of $R_{p}(z)$ is a Cantor set of Lebesgue measure zero. Note that the change $p \mapsto 1-p$ does not change the polynomial $R_{p}(z)$, although the Laplacians $\Delta_{\mu}$ are different.

\section{Spectral zeta function of the Sierpiński Gasket}

We begin this section with describing the analysis on the Sierpiński gasket in the way first developed by J. Kigami [19]. We recall only the basic facts here, and in the papers $4,45,6,11,13,15,16,20,21,24,34,35,36,39$, the reader can find proofs of the propositions given below, as well as the general theory of Dirichlet forms and diffusions on fractals, many physical applications and further references.

If we fix three contractions $F_{j}(x)=\frac{1}{2}\left(x+v_{j}\right)$, where the $v_{j}$ are vertices of a triangle in $\mathbb{R}^{2}$, then the Sierpinski gasket (see Figure 6.1) is a unique compact set $S$ such that

$$
S=F_{1}(S) \cup F_{2}(S) \cup F_{3}(S) .
$$

One can see that the analysis of the Sierpiński gasket is analogous in many respects to the analysis of the fractal Laplacian on an interval we described in Section 5. This analysis does not depend on the particular embedding of the Sierpiński gasket in $\mathbb{R}^{2}$, and so a particular choice of the points $v_{j}$ does not matter as long as they are not collinear.

Definition 6.1. For each $n \geqslant 0$ we define $V_{n}$ by

$$
V_{n+1}=F_{1}\left(V_{n}\right) \cup F_{2}\left(V_{n}\right) \cup F_{3}\left(V_{n}\right),
$$

where $V_{0}=\left\{v_{1}, v_{2}, v_{3}\right\}=\partial S$ is the set of vertices of $S$.

On each $V_{n}$ there is a notion of neighboring points, giving $V_{n}$ a natural graph structure, which can be defined inductively. In Figure 6.2 the neighboring points 


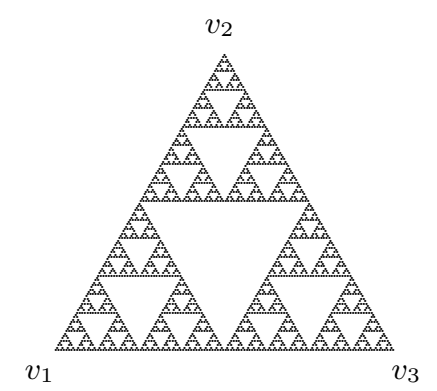

Figure 6.1. Sierpiński gasket
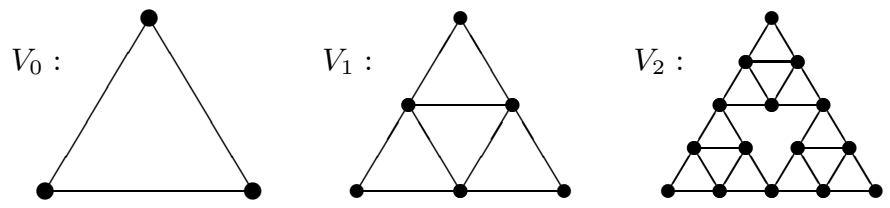

FiguRE 6.2. Discrete approximations to the Sierpiński gasket

are connected by line segments, and in what follows are denoted by the symbol " $x \sim y "$.

Definition 6.2. The discrete Dirichlet (energy) form on $V_{n}$ is

$$
\mathcal{E}_{n}(f, f)=\left(\frac{5}{3}\right)^{n} \sum_{\substack{x, y \in V_{n} \\ y \sim x}}(f(y)-f(x))^{2},
$$

and the Dirichlet (energy) form on $I$ is

$$
\mathcal{E}(f, f)=\lim _{n \rightarrow \infty} \mathcal{E}_{n}(f, f)
$$

if this limit exists and is finite. By the next proposition this limit is increasing and so it always exists as a nonnegative real number or $+\infty$.

A function $h$ is harmonic if it minimizes the energy given the three boundary values.

Proposition 6.3. We have $\mathcal{E}_{n+1}(f, f) \geqslant \mathcal{E}_{n}(f, f)$ for any function $f$, and

$$
\mathcal{E}_{n+1}(h, h)=\mathcal{E}_{n}(h, h)=\mathcal{E}(h, h)
$$

for a harmonic $h$.

Definition 6.4. The discrete Laplacians on $V_{n}$ are

$$
\Delta_{n} f(x)=\frac{1}{4} \sum_{\substack{y \in V_{n} \\ y \sim x}} f(y)-f(x), \quad x \in V_{n} \backslash V_{0},
$$

and the Laplacian on $S$ is

$$
\Delta_{\mu} f(x)=\lim _{n \rightarrow \infty} 5^{n} \Delta_{n} f(x)
$$

if this limit exists and $\Delta_{\mu} f$ is continuous.

The next theorem is essentially due to J. Kigami [19, 20, 21], although many relevant results are contained in [4, 15, 16, 17, 22, 24]. 
Theorem. $\mathcal{E}$ is a local regular Dirichlet form on $S$ which is self-similar in the sense that

$$
\mathcal{E}(f, f)=\frac{5}{3} \sum_{j=1,2,3} \mathcal{E}\left(f \circ F_{j}, f \circ F_{j}\right) .
$$

In particular, the domains of $\mathcal{E}$ and $\Delta_{\mu}$ are contained and dense in the space of continuous functions. The Laplacian satisfies the Gauss-Green (integration by parts) formula:

$$
\mathcal{E}(f, f)=-6 \int_{S} f \Delta_{\mu} f d \mu+\sum_{p \in \partial S} f(p) \partial f(p),
$$

where $\mu$ is the normalized Hausdorff measure, which is self-similar with weights $\frac{1}{3}, \frac{1}{3}, \frac{1}{3}$,

$$
\mu=\frac{1}{3} \sum_{j=1,2,3} \mu \circ F_{j},
$$

and $\partial f(p)$ is a certain derivative at the boundary. The (Dirichlet, Neumann) Laplacian has discrete spectrum and compact resolvent. Green's function $g(x, y)$ is continuous. If $\rho(\lambda)$ is the eigenvalue counting function of the Laplacian $\Delta_{\mu}$, then

$$
0<\liminf _{\lambda \rightarrow \infty} \frac{\rho(\lambda)}{\lambda^{d_{s} / 2}}<\limsup _{\lambda \rightarrow \infty} \frac{\rho(\lambda)}{\lambda^{d_{s} / 2}}<\infty
$$

where the spectral dimension is

$$
1<d_{s}=\frac{\log 9}{\log 5}<2
$$

Next, we present two more theorems illustrating the "fractal" properties of the Laplacian $\Delta_{\mu}$.

Theorem (44,34]). The $\mu$-heat kernel of the Laplacian $\Delta_{\mu}$ (that is, the transition density $p_{t}^{\mu}(x, y)$ of the $\mu$-diffusion) has the non-Gaussian "fractal" asymptotics

$$
p_{t}^{\mu}(x, y) \asymp C t^{-\beta} \exp \left\{-C t^{-\alpha} d_{S}(x, y)^{\gamma}\right\},
$$

where $\alpha=\frac{\log 2}{\log 5-\log 2}, \beta=\frac{\log 3}{\log 5}$ and $\gamma=\frac{\log 5}{\log 5-\log 2}$.

Theorem ([6]). If $f \in \mathcal{D}$ om $\Delta_{\mu}$, then $f^{2} \notin \mathcal{D}$ om $\Delta_{\mu}$ unless $f$ is constant.

Our main result in this section is the following theorem.

Theorem 7. The zeta function of the Laplacian on the Sierpinski gasket is given by

$$
\begin{aligned}
\zeta_{\Delta_{\mu}}(s)=\zeta_{R, \frac{3}{4}}(s) \cdot \frac{5^{-s / 2}}{2} \cdot\left(\frac{1}{1-3 \cdot 5^{-s / 2}}\right. & \left.+\frac{3}{1-5^{-s / 2}}\right) \\
& +\zeta_{R, \frac{5}{4}}(s) \cdot \frac{5^{-s}}{2} \cdot\left(\frac{3}{1-3 \cdot 5^{-s / 2}}-\frac{1}{1-5^{-s / 2}}\right),
\end{aligned}
$$

where $R(z)=z(5-4 z)$. In particular, $\zeta_{\Delta_{\mu}}(s)$ has a meromorphic continuation for $\operatorname{Re}(s)>-\varepsilon$, where $\varepsilon \geqslant 1$. The poles are contained in

$$
\left\{\frac{2 i n \pi}{\log 5}, \frac{\log 9+2 i n \pi}{\log 5}: n \in \mathbb{Z}\right\} \text {. }
$$

We have $0<\frac{\log 4}{\log 5}=d_{R}<1<d_{s}=\frac{\log 9}{\log 5}<2$. 


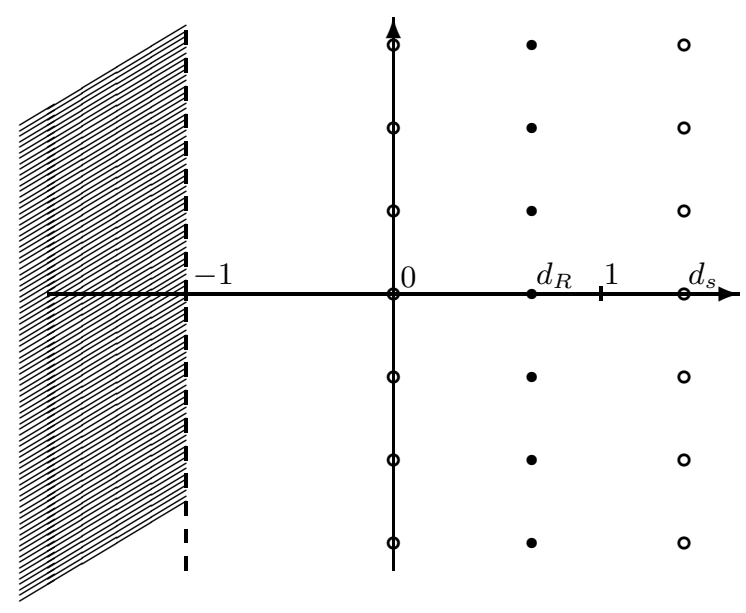

FiguRE 6.3. Complex spectral dimensions of the Laplacian on the Sierpiński gasket

Proof. By [16, 39], every eigenvalue of $\Delta_{\mu}$ has the form

$$
\lambda=-5^{m} \lim _{n \rightarrow \infty} 5^{n} R^{-n}\left(z_{0}\right),
$$

where $R^{-n}\left(z_{0}\right)$ is a preimage of $z_{0}=3 / 4,5 / 4$ under the $n$-th iteration power of the polynomial $R(z)$. The multiplicity of such an eigenvalue is of the form $C_{1} 3^{m}+C_{2}$, where $C_{1}$ and $C_{2}$ are computed explicitly in [16, 39. More precisely, by [39, Proposition 3.12], for the discrete Laplacian we have that the multiplicity of $z \in R^{-m}\left(\frac{3}{4}\right)$ is $\frac{3^{n-m-1}+3}{2}$ for $n \geqslant 1$ and $0 \leqslant m \leqslant n-1$, and the multiplicity of $z \in R^{-m}\left(\frac{5}{4}\right)$ is $\frac{3^{n-m-1}-1}{2}$ for $n \geqslant 2$ and $0 \leqslant m \leqslant n-2$. Then the proof follows directly from Theorem 2 and the definition of the zeta function of a polynomial.

Remark 6.5. We have that the zeros of the "geometric part" cancel all the poles of the zeta function of the polynomial in the product formula (6.1). We conjecture this also for other symmetric p.c.f. fractals [33, except one dimensional. In Figure 6.3 the series of the canceled poles is denoted by black dots, and the other two series of poles are denoted by small circles.

Remark 6.6. Note that the zeta function of the polynomial in (6.1) also describes the distribution of the eigenvalues corresponding to the nonlocalized eigenfunctions (see [3, 39]).

Remark 6.7. The method of Theorem 7 applies to a large class of fractals (see, for instance, [25, 33, 39, 40]), and possibly to [37.

\section{ACKNOWLEDGMENT}

The author is deeply grateful to M. Lapidus for mentoring, encouragement and support, and to R. Strichartz and J. Kigami for many helpful discussions. 


\section{REFERENCES}

[1] B. Adams, S.A. Smith, R. Strichartz and A. Teplyaev, The spectrum of the Laplacian on the pentagasket. Fractals in Graz 2001 - Analysis - Dynamics - Geometry - Stochastics, 1-24, Trends Math., Birkhäuser Basel (2003). MR2091699 (2006g:28017)

[2] M. T. Barlow, Diffusions on fractals. Lectures on Probability Theory and Statistics (SaintFlour, 1995), 1-121, Lecture Notes in Math., 1690, Springer, Berlin, 1998. MR.1668115 (2000a:60148)

[3] M. T. Barlow and J. Kigami, Localized eigenfunctions of the Laplacian on p.c.f. self-similar sets, J. London Math. Soc., 56 (1997), 320-332. MR.1489140 (99b:35162)

[4] M.T. Barlow and E.A. Perkins, Brownian motion on the Sierpiński gasket. Probab. Theory Related Fields 79 (1988), 543-623. MR0966175 (89g:60241)

[5] M. F. Barnsley, J. S. Geronimo and A. N. Harrington, Condensed Julia sets, with an application to a fractal lattice model Hamiltonian. Trans. Amer. Math. Soc. 288 (1985), 537-561. MR:0776392 (86h:58088)

[6] O. Ben-Bassat, R. S. Strichartz and A. Teplyaev, What is not in the domain of the Laplacian on Sierpiński gasket type fractals. J. Funct. Anal., 166 (1999), 197-217. MR1707752 (2001e:31016)

[7] D. Bessis, J. Geronimo and P. Moussa, Mellin transforms associated with Julia sets and physical applications, J. Statist. Phys. 34 (1984), 75-110. MR.0739123 (85i:58067)

[8] D. Bessis, J. Geronimo and P. Moussa, Function weighted measures and orthogonal polynomials on Julia sets, Constr. Approx. 4 (1988), 157-173. Erratum, Constr. Approx. 6 (1990), 335-336. MR0932652 (89h:58085) MR.1054759 (91b:58210)

[9] E.J. Bird, S.-M. Ngai and A. Teplyaev, Fractal Laplacians on the Unit Interval, Ann. Sci. Math. Québec 27 (2003), 135-168. MR2103098 (2006b:34192)

[10] H. Brolin, Invariant sets under iteration of rational functions, Ark. Mat. 6 (1965), 103-144. MR0194595 (33:2805)

[11] K. Dalrymple, R. S. Strichartz and J. P. Vinson, Fractal differential equations on the Sierpinski gasket. J. Fourier Anal. Appl., 5 (1999), 203-284. MR.1683211 (2000k:31016)

[12] G. Derfel, P. Grabner and F. Vogl, The zeta function of the Laplacian on certain fractals, to appear in Trans. Amer. Math. Soc.

[13] E. Domany, S. Alexander, D. Bensimon and L. Kadanoff, Solutions to the Schrödinger equation on some fractal lattices. Phys. Rev. B (3) 28 (1984), 3110-3123. MR0717348 (85h:82033)

[14] P. Fatou, Sur les équations fonctionnelles. Bull. Soc. Math. France, 47 (1919), 161-271; 48 (1920), 33-94, 208-314. MR.1504787 MR1504792 MR1504797

[15] M. Fukushima, Dirichlet forms, diffusion processes and spectral dimensions for nested fractals. Ideas and methods in Mathematical Analysis, Stochastics, and applications (Oslo, 1988), 151-161, Cambridge Univ. Press, Cambridge, 1992. MR1190496 (94d:60129)

[16] M. Fukushima and T. Shima, On a spectral analysis for the Sierpinski gasket. Potential Analysis 1 (1992), 1-35. MR1245223 (95b:31009)

[17] S. Goldstein, Random walks and diffusions on fractals, in "Percolation Theory and Ergodic Theory of Infinite Particle Systems" (H. Kesten, ed.), 121-129, IMA Math. Appl., Vol. 8, Springer, New York, 1987. MR0894545 (88g:60245)

[18] P. Grabner, Functional iterations and stopping times for Brownian motion on the Sierpiński gasket. Mathematika 44 (1997), 374-400. MR1600494 (99b:60128)

[19] J. Kigami, A harmonic calculus on the Sierpinsski spaces. Japan J. Appl. Math. 6 (1989), 259-290. MR:1001286 (91g:31005)

[20] J. Kigami, Harmonic calculus on p.c.f. self-similar sets. Trans. Amer. Math. Soc. 335 (1993), 721-755. MR1076617 (93d:39008)

[21] J. Kigami, Analysis on fractals. Cambridge Tracts in Mathematics 143, Cambridge University Press, 2001. MR1840042(2002c:28015)

[22] J. Kigami and M. L. Lapidus, Weyl's problem for the spectral distribution of Laplacians on p.c.f. self-similar fractals. Comm. Math. Phys. 158 (1993), 93-125. MR1243717 (94m:58225)

[23] J. Kigami and M. L. Lapidus, Self-similarity of volume measures for Laplacians on p.c.f. self-similar fractals. Comm. Math. Phys. 217 (2001), 165-180. MR1815029 (2002j:35237) 
[24] S. Kozlov, Harmonization and homogenization on fractals. Comm. Math. Phys. 153 (1993), 339-357. MR1218305 (94c:35026)

[25] B. Krön and E. Teufl, Asymptotics of the transition probabilities of the simple random walk on self-similar graphs, Trans. Amer. Math. Soc., 356 (2003) 393-414. MR 2020038 (2004k:60130)

[26] M. L. Lapidus, Spectral and fractal geometry: From the Weyl-Berry conjecture for the vibrations of fractal drums to the Riemann zeta-function. Differential equations and mathematical physics (Birmingham, AL, 1990), 151-181, Math. Sci. Engrg., 186, Academic Press, Boston, MA, 1992. MR.1126694 (93f:58239)

[27] M. L. Lapidus and M. van Frankenhuysen, Fractal Geometry and Number Theory. Complex Dimensions of Fractal Strings and Zeros of Zeta Functions. Birkhäuser, Boston, 2000. MR:1726744 (2001b:11079)

[28] M. L. Lapidus and M. van Frankenhuysen, Fractality, self-similarity and complex dimensions. Fractal Geometry and Applications: A Jubilee of Benoit Mandelbrot, Part 1. Proceedings of Symposia in Pure Mathematics 72, Amer. Math. Soc., (2004), 349-372. MR2112111 (2005k:11142)

[29] M. L. Lapidus and H. Maier, Hypothèse de Riemann, cordes fractales vibrantes et conjecture de Weyl-Berry modifiée. Acad. Sci. Paris Sér. I Math. 313, (1991), 19-24. MR1115940 (92f:11118)

[30] M. L. Lapidus and H. Maier, The Riemann hypothesis and inverse spectral problems for fractal strings. J. London Math. Soc. (2) 52, (1995), 15-34. MR.1345711 (97b:11111)

[31] M. L. Lapidus and C. Pomerance, Fonction zêta de Riemann et conjecture de Weyl-Berry pour les tambours fractals. C. R. Acad. Sci. Paris Sér. I Math. 310, (1990), 343-348. MR 1046509 (91d:58248)

[32] M. L. Lapidus and C. Pomerance, The Riemann zeta-function and the one-dimensional Weyl-Berry conjecture for fractal drums. Proc. London Math. Soc. (3) 66, (1993), 41-69. MR.1189091 (93k:58217)

[33] L. Malozemov and A. Teplyaev, Self-similarity, operators and dynamics. Math. Phys. Anal. Geom. 6 (2003), 201-218. MR1997913 (2004d:47012)

[34] V. Metz and K.-T. Sturm, Gaussian and non-Gaussian estimates for heat kernels on the Sierpiński gasket. Dirichlet forms and stochastic processes (Beijing, 1993), 283-289, de Gruyter,Berlin, 1995. MR1366443 (97b:60132)

[35] R. Rammal, Spectrum of harmonic excitations on fractals. J. Physique 45 (1984), 191-206. MR $0737523(85 \mathrm{~d}: 82101)$

[36] R. Rammal and G. Toulouse, Random walks on fractal structures and percolation clusters. J. Physique Letters 44 (1983), L13-L22.

[37] C. Sabot, Electrical networks, symplectic reductions, and application to the renormalization map of self-similar lattices. J. Physique Letters 44 (1983), L13-L22. Fractal Geometry and Applications: A Jubilee of Benoit Mandelbrot, Part 1. Proceedings of Symposia in Pure Mathematics 72, Amer. Math. Soc., (2004), 155-205. MR2112106 (2005m:34202)

[38] T. Shima, On eigenvalue problems for Laplacians on p.c.f. self-similar sets, Japan J. Indust. Appl. Math. 13 (1996), 1-23. MR.1377456 (97f:28028)

[39] A. Teplyaev, Spectral Analysis on Infinite Sierpiński Gaskets, J. Funct. Anal., 159 (1998), 537-567. MR.1658094 (99j:35153)

[40] A. Teplyaev, Spectral zeta function of symmetric Sierpiński gasket type fractals, Fractal Geometry and Stochastics III, Progress in Probability 57, Birkhäuser (2004), 245-262. MR:2087144 (2005h:28028)

Department of Mathematics, University of Connecticut, Storrs, Connecticut 06269

E-mail address: teplyaev@math.uconn.edu 